Article

\title{
Power Transformer Insulation Assessment based on Oil-Paper Measurement Data using SVM-Classifier
}

\author{
Suwarno ${ }^{1 *}$, Rahman A. Prasojo ${ }^{1}$ \\ 1 School of Electrical Engineering and Informatics, Institut Teknologi Bandung, Bandung 40132, Indonesia. \\ * Correspondence: suwarno@stei.itb.ac.id;
}

\begin{abstract}
Oil immersed paper insulation condition is a crucial aspect of power transformer's life condition diagnostic. The measurement testing database collected over the years made it possible for researchers to implement classification analysis to in-service power transformer. This article presents classification analysis of transformer oil-immersed paper insulation condition. The measurements data (dielectric characteristics, dissolved gas analysis, and furanic compounds) of 149 transformers with primary voltage of $150 \mathrm{kV}$ had been gathered and analyzed. The algorithm used for developing classification model is Support Vector Machine (SVM). The model has been trained and tested using different datasets. Different models have been created and the best chosen, resulting in $90.63 \%$ accuracy in predicting the oil-immersed paper insulation condition. Further implementation was executed to classify oil-paper condition of 19 Transformers which Furan data is not available. The classification results combined, reviewed, and compared to conventional assessment methods and standards, confirming that the model developed has the ability to do classification of current oil-paper condition based on Dissolved Gasses and Dielectric Characteristics.
\end{abstract}

Keywords: support vector machine; classification analysis; power transformer condition assessment; oil immersed paper insulation; dga; dielectric characteristics, furanic compounds

\section{Introduction}

Oil-impregnated paper is commonly used insulator in power transformers. Evaluation of the degradation of transformer paper insulation in an oil-filled transformer is critical due to the importance of power transformer in the electrical supply chain. Whilst monitoring condition of oil insulation can be done easily, assessing the state of paper insulation is more difficult because the paper is wrapped around the conductors and cannot be sampled without taking the transformer out of service [1]. Different diagnostic method using Dissolved Gas Analysis (DGA) and aging estimation from loading history has been used. The application of 2FAL (2-furaldehyde) as measurement of specific chemical indicator of the aging of paper insulation has received increased attention in the last 20 years [2].

The degradation of cellulose paper insulation in oil-filled power transformer is promoted by four agents of degradation, such as, exposure to elevated temperature, oxygen, acid, and moisture. The processes of degradation for this are thermal, oxidation, and hydrolysis. These degradations caused chain scission or depolymerization and decreasing the tensile strength of paper, yielding glucose. This glucose will further degrade to form furans and other chemical products such as water and gases. The advantage of furan assessment, is that the it does not produce by oil degradation.

Despite the fact that furan is the most accessible yet reliable transformer paper assessment, this measurement is not done periodically by the utility. In order to find out the current condition of paper insulation, it is needed another inexpensive approach. Machine learning algorithm can be employed to model the current transformer paper condition level. Several studies have been done to figure out the possibility of this method. Transformer remnant life prediction using furan in [3] used Fuzzy Logic. K-NN and Decision Tree based classification for prediction of transformer furan level [4]. ANFIS was used to predict the Degree of Polymerization and further do the expected life 
estimation of transformer in [5], and simple multiple regression model has also developed to be compared with ANFIS model in [6].

SVM is one of the commonly adopted machine learning algorithms for data classification [7] [8] [9]. SVM used in [10] to forecast electric load along with other algorithm such as Fuzzy Time Series and Global Harmony Search. A computational model was developed to estimate mass concentration of boiler flue gas in [11]. Study in [12] implemented SVM to classify the results of the simulation in defining synchronization capability limits of permanent-magnet motor. In power transformer diagnosis, SVM was implemented in [13] [14] [15] and [16] for fault detection. Several machine learning approach were used in [17], SVM was utilized along with Decision Tree, ANN, KNN, and Naïve Bayes to assess transformer furan content. This publication comes out with relatively low accuracy on SVM classifier.

There is no single scientific method available to determine the condition or end-of-life of an operating power transformer, the combination of analytical, inspection and testing methods, when used together help form a complete picture of the condition of units in service [18].

This article will implement classification analysis using SVM as an additional insight to help utilities assess transformer oil-impregnated paper insulation condition by using transformer oil measurements data. The main issues of developing SVM model classification analysis will be discussed, which are, data preparation, feature selection, and model validation with different models to find the best model created in order to meet accuracy level intended. The proposed model then compared to conventional methods and standards to validate the classification result.

\section{Methodology}

This section presents the step by step methodology of SVM classifier model development. The attributes observed, guidelines of 2FAL assessment, model development flowchart, preprocessing and outlier elimination, and SVM classifier algorithm is presented in this section.

\subsection{Sample}

Measurements data (dissolved gases, oil dielectric characteristics, and furan) of 149 in-service transformers has been gathered. Figure 1 shows one of the transformers observed in this study. All of the measurements data are from 3 phase power transformers with $150 \mathrm{kV}$ primary voltage, and operating life of 3 up to 44 years. For the detail data

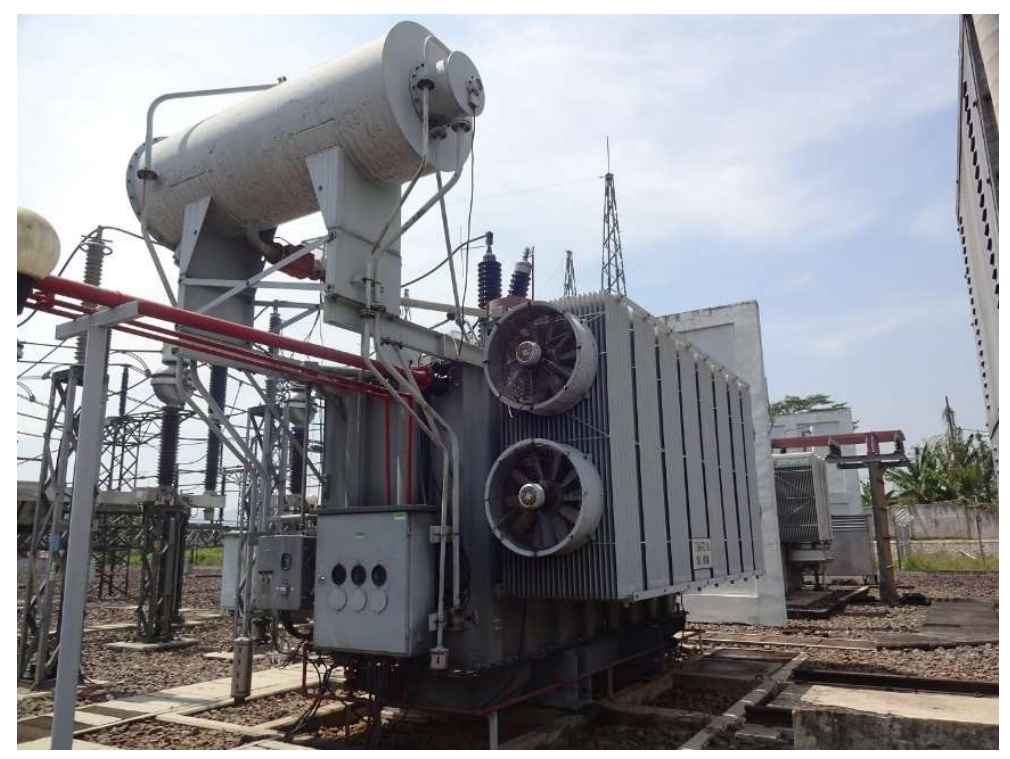

Figure 1. A sample of $150 \mathrm{kV}$ power transformer used in this study

\subsection{Attributes Observed}


In this article, the authors gathered measurements data consisting of dissolved gases, dielectric characteristics, and furanic compounds.

\subsubsection{Dissolved Gas Analysis}

DGA of insulating oil is universally used and considered as an important indicator of transformer's overall condition all over the world [19]. The dissolved gasses were measured and interpreted based on [20], consists of Hydrogen (H2), Methane (CH4), Carbon Monoxide (CO), Carbon Dioxide $(\mathrm{CO} 2)$, Ethylene $(\mathrm{C} 2 \mathrm{H} 4)$, Ethane $(\mathrm{C} 2 \mathrm{H} 6)$, Acetylene $(\mathrm{C} 2 \mathrm{H} 2)$ and Total Dissolved Combustible Gas (TDCG).

\subsubsection{Dielectric Characteristics}

Characteristics of transformer oil insulation were measured and interpreted based on [21], consists of Breakdown Voltage in kV (IEC 60156), Water Content in ppm (IEC 60814), Acidity in mg $\mathrm{KOH} / \mathrm{g}$ (IEC 62021), Interfacial Tension in dyne/cm (ASTM D971), and Color Scale (ISO 2049).

\subsubsection{Furanic Compounds}

Furans are part of the degradation products of cellulose insulation paper in transformers, and they are partially soluble in the insulation fluid [2]. Most often, five furanic compounds measured are 2-furaldehyde (2FAL), 5-methyl-2-furaldehyde (5M2F), 5-hydroxymethyl-2-furaldehyde (5H2F), 2-acetyl furan (2ACF), and 2-furfurol (2FOL). 2FAL is considered as the main compound among these furanic compounds because of its higher generation rate and stability inside a transformer. 2FAL is usually correlated to Degree of Polymerization (DP). Paper with initial DP value of approximately 1000 is expected to last the lifetime of the transformer (25-30 years), but a DP of 150-250 is regarded to be the end of life criterion for the transformer insulation because the paper is also at risk of mechanical failure [22].

\subsection{Analysis Methods}

2FAL is the most accessible measurement for assessing insulation paper of power transformer, however, 2FAL is not a routine test. This subsection discussed the methods of assessing oil-immersed paper in power transformer when there are furan measurements and using SVM-classifier when no furan measurement is available.

\subsubsection{Determining Oil-Paper Condition based on Measurement Data}

Table 1 shows the guidelines used for assessing the significance of 2FAL measurement, as used by several publications [23] [3] [24]. The correlation between 2FAL and Degree of Polymerization with its extent of degradation is shown. Measurement data of 2FAL falls into categories in Table 1, 'Healthy', 'Moderate', 'Extensive', and 'End of Life'. When degree of polymerization of transformer paper reach the value of 250 or lower, the paper considered to lost its mechanical strength and transformer has reached its end of life. Table 2 and Figure 3 shows the number of transformers measurement data that falls into each category.

Table 1. Guidelines for Oil Immersed Insulation Paper Degradation

\begin{tabular}{ccc}
\hline 2FAL (ppm) & DP Value & Oil-Paper Health Category \\
\hline $0-0.1$ & $1200-700$ & Healthy Insulation \\
$0.1-1$ & $700-450$ & Moderate Deterioration \\
$1-10$ & $450-250$ & Extensive Deterioration \\
$>10$ & $<250$ & End of Life Criteria \\
\hline
\end{tabular}

Table 2. Number of data each category 


\begin{tabular}{ccc}
\hline Category & $\begin{array}{c}\text { Transformer Number in } \\
\text { Corresponding Category }\end{array}$ & Percentage \\
\hline Healthy Insulation & 67 & $48.9 \%$ \\
Moderate Deterioration & 54 & $39.42 \%$ \\
Extensive Deterioration & 16 & $11,67 \%$ \\
\hline
\end{tabular}

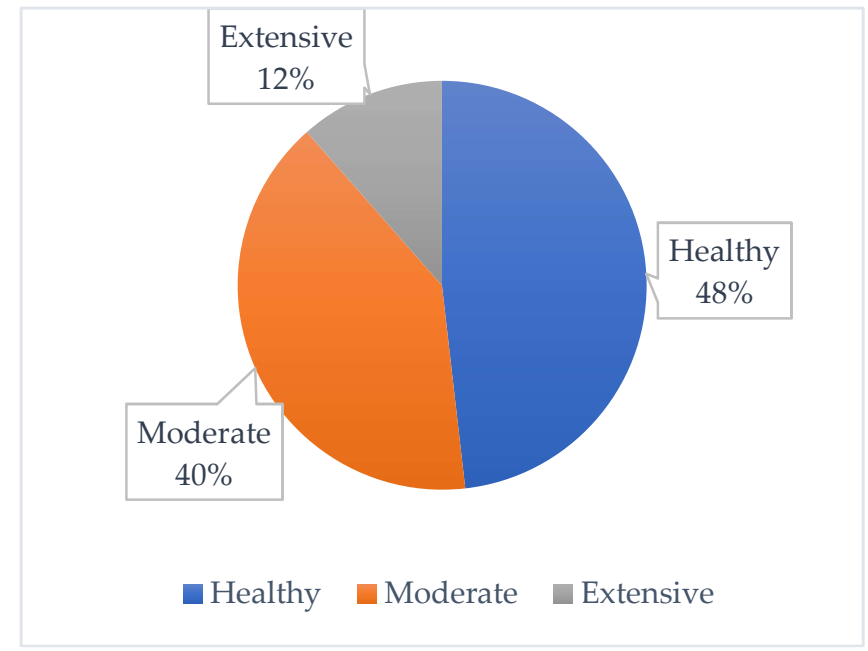

Figure 2. Percentage of Data each Category

\subsubsection{Support Vector Machine Classifier}

Support Vector Machine (SVM) is an efficient algorithm in learning theory, especially for classification problems. The classic svm was introduced with polynomial kernels by Boser et al. in [25], and with general kernels by Cortes and Vapnik in [26]. Among other linear programming, SVM is important because of its linearity and flexibility for large data setting [27]. SVM is a powerful supervised learning algorithm, which is based on learning algorithm related to data analysis for classification and regression analysis. SVM is known to be efficient, particularly in large classification problems, because the training of the classified vectors does not have a distinct influence on the performance of SVM. Therefore, SVM has the required potential to handle very large feature spaces. Also, SVM-based classifiers are claimed to have good generalization properties compared with conventional classifiers, because in training the SVM classifier, the structural misclassification risk is to be minimized, whereas traditional classifiers are usually trained so that empirical risk is minimized [15].

SVM is one of the standard algorithm for data mining and machine learning based on the advances theory of statistical learning. Various different binary classification methods are implemented for the purpose of multi-category classification, such as 'one-against-all', 'one-against-one', etc [28]. Linear programming SVM classifier is especially efficient for very large size samples. But little is known about its convergence, compared with the well understood quadratic programming SVM classifier [27]. This study will compare the classification accuracy of both linear and quadratic SVM classifier. The three classifications shown in Table 1, 'Healthy', Moderate', and 'Extensive' will be target category for SVM classifier. 'End of life' category was not included in this discussion due to no transformer measurement data collected was included in that category. The SVM model developed will be linear and quadratic SVM. Linear programming means the algorithm is based on linear programming optimization, while Quadratic programming means it is based on quadratic programming optimization [29]. The difference between Linear and Quadratic SVM is thoroughly discussed in [27]. Vapnik in [29] introduced Linear SVM algorithm associated to a Mercer Kernel K. It is based on the following linear programming optimization problem in equation 1. 


$$
\begin{gathered}
\min _{\alpha \in R_{+}^{m}, b \in R}\left\{\frac{1}{m} \sum_{i=1}^{m} \xi_{i}+\frac{1}{C} \sum_{i=1}^{m} \alpha_{i}\right\} \\
\text { subject to } \quad y_{i}\left(\sum_{j=1}^{m} \alpha_{j} y_{j} K\left(x_{i}, x_{j}\right)+b\right) \geq 1-\xi_{i} \\
\xi_{i} \geq=, i=1, \ldots, m .
\end{gathered}
$$

Here $\alpha=\left(\alpha_{1}, \cdots, \alpha_{m}\right)$, $\xi$ i's are slack variables. The trade-off parameter $\mathrm{C}=\mathrm{C}(\mathrm{m})>0$ depends on $\mathrm{m}$ and is crucial. If $\left(\alpha_{z}=\left(\alpha_{1, z}, \ldots, \alpha_{m, z}\right), \mathrm{b}_{z}\right)$ solves the optimization problem (equation 1$)$, the Linear-SVM classifier is given by sgn $\left(f_{z}\right)$ with equation 2 .

$$
f_{z}(x)=\sum_{i=1}^{m} \alpha_{i}, z y_{j} K\left(x, x_{i}\right)+b_{z}
$$

For a real-valued function $f: X \rightarrow R$, its sign function is defined as $\operatorname{sgn}(f)(x)=1$ if $f(x) \geq 0$ and $\operatorname{sgn}(\mathrm{f})(\mathrm{x})=-1$ otherwise. The Quadratic SVM is based on a quadratic programming optimization problem in equation 3.

$$
\begin{gathered}
\min _{\alpha \in R_{+}^{m}, b \in R}\left\{\frac{1}{m} \sum_{i=1}^{m} \xi_{i}+\frac{1}{2 \tilde{C}} \sum_{i, j=1}^{m} \alpha_{i} y_{i} K\left(x_{i}, x_{j}\right) \alpha_{j} y_{j}\right\} \\
\text { subject to } \quad y_{i}\left(\sum_{j=1}^{m} \alpha_{j} y_{j} K\left(x_{i}, x_{j}\right)+b\right) \geq 1-\xi_{i} \\
\xi_{i} \geq=, i=1, \ldots, m .
\end{gathered}
$$

Here $\tilde{C}=\tilde{C}(m)>0$ is also a trade-off parameter depending on the sample size $\mathrm{m}$. If $\left(\tilde{\alpha}_{z}=\right.$ $\left.\left(\tilde{\alpha}_{1, z}, \ldots, \tilde{\alpha}_{m, z}\right), \tilde{b}_{z}\right)$ solves the optimization problem (equation 3 ), then the 1-norm soft margin classifier is defined by $\operatorname{sgn}\left(\widetilde{f}_{z}\right)$ with

$$
f_{z}(x)=\left(\sum_{i=1}^{m} \tilde{\alpha}_{i}, z y_{j} K\left(x, x_{i}\right)+\tilde{b}_{z}\right)
$$

\subsection{Classification Model Flowchart}

Figure 3 shows the process of developing classification analysis in this study. First, measurements data were accessed and explored. These data including of Transformer Profile, Dissolved Gasses, Dielectric Characteristics, and Furanic Compound. Then, the data from different sources will be composed to the same format. The outliers were eliminated using one-class SVM. The inliers data was separated to training and testing datasets.

\section{Results and Discussion}

This section presents the results of SVM model development in classification analysis of transformer paper insulation condition. In this section, the data preparation, classification result, and model validation are presented.

\subsection{Data Preprocessing}

Measurement data gathered to develop classification model consist of dielectric characteristics and dissolved gasses with total 15 attributes. Before developing the model, the attributes are ranked by ANOVA and chi-squared criteria:

- Analysis of variance (ANOVA): the difference between average values of the feature in different classes, in order to find out if an attribute is significant for model development. 


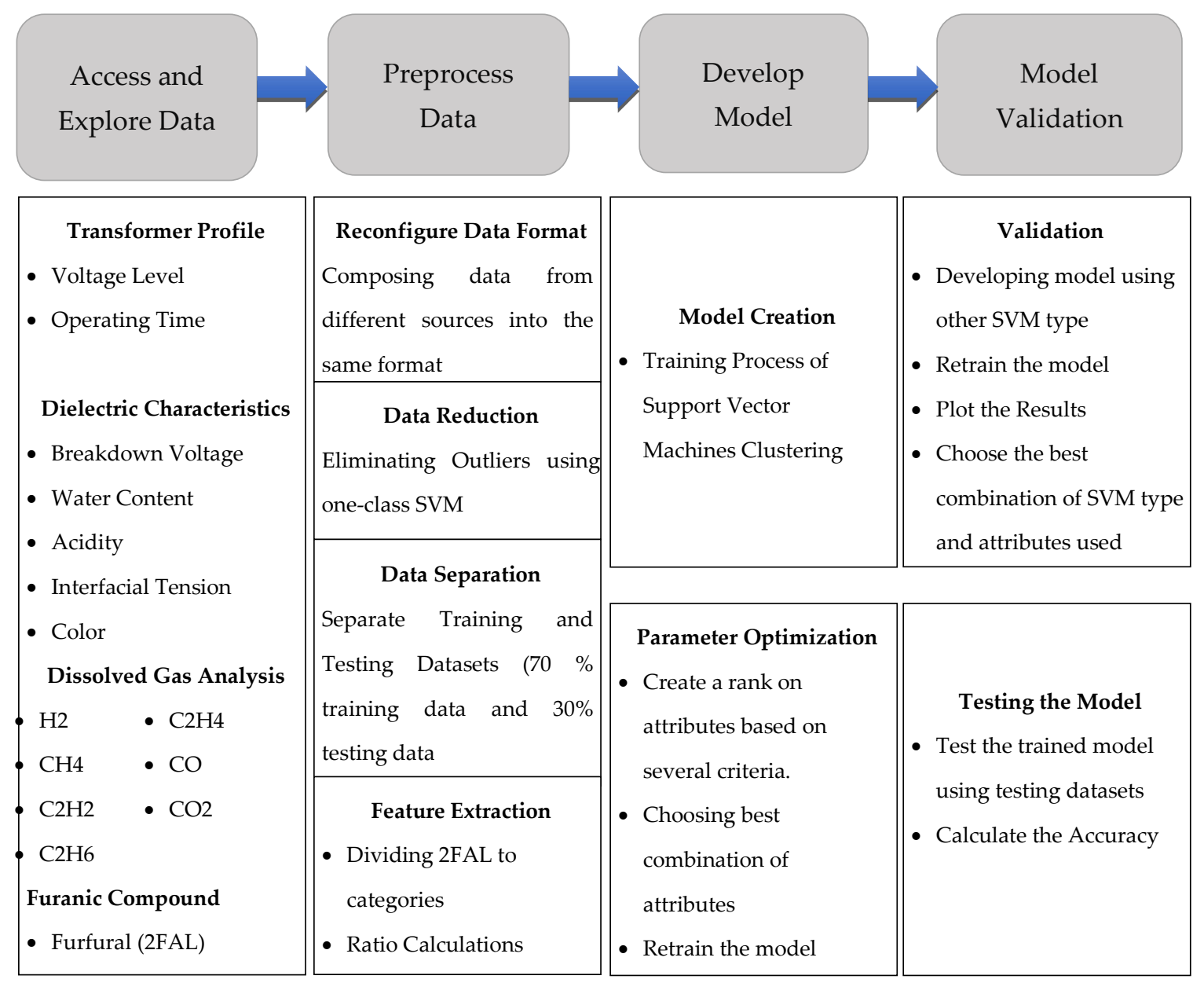

Figure 3. Classification model development flowchart

Steps for ANOVA calculations [30].

a. Calculate the correction factor using equation 5 .

$$
C F=\frac{\left(\sum x\right)^{2}}{N}
$$

b. Calculate the sum of squares total value (SS Total) using equation 6 .

$$
\text { SS Total }=\sum x^{2}-C F
$$

c. Equation 7 to calculate the SS Group value.

$$
S S \text { Group }=\sum \frac{\left(\sum x\right)^{2}}{n}-C F
$$

d. Equation 8 to do calculation of the SS error value.

$$
\text { SS Error = SS Total }- \text { SS Group }
$$

e. Calculate MS group value using equation 9 .

$$
\text { MS Group }=\frac{\text { SS Group }}{d f \text { Group }}
$$

f. Calculate MS error value using equation 10 .

$$
M S \text { Error }=\frac{S S \text { Error }}{d f \text { Error }}
$$

g. Equation 11 to calculate Variance Ratio (V.R.)

$$
V . R .=\frac{M S \text { Group }}{M S \text { Error }}
$$

- Chi-squared: dependence between the feature and the class as measure by the chi-square statistic, the calculation is done using equation 8 . 


$$
x^{2}=\sum_{i=1}^{n} \frac{\left(O_{i}-E_{i}\right)^{2}}{E_{i}}
$$

$x^{2}=$ Pearson's cumulative test statistic

$O_{i}=$ the number of observations of type $i$.

$E_{i}=N_{p i}=$ the expected (theoretical) frequency of type $i$, asserted by the null hypothesis that the fraction of type $i$ in the population is $p_{i}$

$n=$ the number of cells in the table.

Table 3. Rank of attributes based on ANOVA and chi-squared

\begin{tabular}{cccc}
\hline Rank & Attributes & ANOVA & Chi-squared \\
\hline 1 & Color & 44.16 & 36.72 \\
\hline 2 & IFT & 19.87 & 19.95 \\
\hline 3 & CO & 15.04 & 17.87 \\
\hline 4 & CO2 & 11.63 & 16.79 \\
\hline 5 & CO+CO2 & 13.92 & 15.42 \\
\hline 6 & TDCG & 1.24 & 9.09 \\
\hline 7 & Acidity & 9.82 & 8.24 \\
\hline 8 & C2H2 & 0.15 & 4.52 \\
\hline 9 & H2 & 0.56 & 3.68 \\
\hline 10 & Water Content & 1.17 & 2.87 \\
\hline 11 & C2H6 & 0.63 & 2.66 \\
\hline 12 & C2H4 & 0.50 & 2.36 \\
\hline 13 & CO2/CO & 1.20 & 1.27 \\
\hline 14 & Breakdown Voltage & 0.20 & 0.76 \\
\hline 15 & CH4 & 0.01 & 0.32 \\
\hline
\end{tabular}

Table 3 shows the rank of attributes based on ANOVA and chi-squared. Color has the highest ANOVA and chi-square, followed by IFT, CO, CO2, accumulation of CO+CO2, TDCG, acidity, and other attributes. This rank is then used for attributes selection in SVM model development.

\subsection{Data Reduction: Eliminating Outliers}

As much as 149 transformer measurements data collected, were analyzed under Orange Data Mining Program to find the outliers using one-class SVM with non-linear kernel (RBF). This is an unsupervised learning algorithm that learns a decision function for novelty detection. It classifies new data as similar or different to the training set [31]. The inliers data from this process (102 data) were used as SVM model development and validation.

\subsection{Dataset Preparation}

Table 4. Training and testing data separation

\begin{tabular}{ccc}
\hline Datasets & Category & $\begin{array}{c}\text { Number of } \\
\text { Transformers }\end{array}$ \\
\hline Training & Healthy & 37 \\
(70 Transformers) & Moderate & 27 \\
& Extensive & 6 \\
\hline \multirow{2}{*}{ Testing } & Healthy & 17 \\
(32 Transformers) & Moderate & 12 \\
& Extensive & 3 \\
\hline
\end{tabular}


The inliers data resulted from outlier elimination then divided into two datasets. of $150 \mathrm{kV}$ transformer testing measurements were collected. These data consist of three paper condition categories as shown in Table 2 and Figure 2. There are 54 transformers in 'Healthy' category, 39 transformers in 'Moderate' category, and 9 transformers in 'Extensive' category.

The measurements data then divided into two datasets, with 70 transformers in training datasets and 32 transformers in testing datasets. The configuration of training and testing data is shown in Table 4.

\subsection{SVM Classification Model Development}

Three categories of transformer paper degradation level, 'Healthy', 'Moderate', and 'Extensive' were used as target class. The attributes included were dissolved gasses and dielectric characteristics, with the total of 15 . The attributes selection is shown in Table 5 . The attributes selection is based on the rank discussed in subsection 3.1.

Table 5. Attributes Selection

\begin{tabular}{|c|c|c|c|c|c|c|}
\hline Parameters No. of Attributes & 15 & 8 & 7 & 6 & 5 & 4 \\
\hline $\mathrm{H} 2$ & o & $x$ & $x$ & $x$ & $\mathrm{x}$ & $x$ \\
\hline $\mathrm{CH} 4$ & $\mathrm{o}$ & $x$ & $x$ & $x$ & $x$ & $x$ \\
\hline $\mathrm{C} 2 \mathrm{H} 4$ & o & $x$ & $x$ & $x$ & $x$ & $x$ \\
\hline $\mathrm{C} 2 \mathrm{H} 6$ & o & $x$ & $x$ & $x$ & $x$ & $\mathrm{x}$ \\
\hline $\mathrm{C} 2 \mathrm{H} 2$ & o & o & o & $x$ & $x$ & $x$ \\
\hline TDCG & $\mathrm{o}$ & $\mathrm{o}$ & o & $\mathrm{o}$ & $x$ & $\mathrm{x}$ \\
\hline Water & $\mathrm{o}$ & $x$ & $x$ & $x$ & $x$ & $\mathrm{x}$ \\
\hline BDV & o & $x$ & $x$ & $x$ & $x$ & $\mathrm{x}$ \\
\hline $\mathrm{CO}$ & o & o & o & o & o & o \\
\hline $\mathrm{CO} 2$ & o & o & o & o & $\mathrm{o}$ & o \\
\hline $\mathrm{CO}+\mathrm{CO} 2$ & o & o & $x$ & $x$ & $x$ & $x$ \\
\hline $\mathrm{CO} 2 / \mathrm{CO}$ & $\mathrm{o}$ & $\mathrm{x}$ & $\mathrm{x}$ & $\mathrm{x}$ & $\mathrm{x}$ & $x$ \\
\hline Acidity & o & o & o & o & $\mathrm{o}$ & $\mathrm{x}$ \\
\hline IFT & $\mathrm{o}$ & $\mathrm{O}$ & $\mathrm{O}$ & $\mathrm{o}$ & $\mathrm{o}$ & $\mathrm{O}$ \\
\hline Color & o & o & $\mathrm{O}$ & o & o & o \\
\hline
\end{tabular}

Table 6 shows 12 models created using Linear and Quadratic SVM. Training and testing datasets were used to evaluate the model, with respective accuracy. The best-chosen model was number 12, with attributes of CO, CO2, IFT, and Color. This model was able to do classification of testing dataset with $90.63 \%$ accuracy.

Table 6. Accuracy of different SVM models

\begin{tabular}{ccccc}
\hline & Training & Testing & & $\begin{array}{c}\text { No. of } \\
\text { No. }\end{array}$ \\
Accuracy & Accuracy & Type & Features \\
\hline 1 & 85.71 & 81.25 & Linear & 15 \\
\hline 2 & 94.29 & 75.00 & Quadratic & 15 \\
\hline 3 & 82.86 & 81.25 & Linear & 8 \\
\hline 4 & 92.86 & 78.13 & Quadratic & 8 \\
\hline 5 & 82.86 & 81.25 & Linear & 7 \\
\hline 6 & 92.86 & 78.13 & Quadratic & 7
\end{tabular}




\begin{tabular}{ccccc}
7 & 80.00 & 84.38 & Linear & 6 \\
\hline 8 & 91.43 & 71.88 & Quadratic & 6 \\
\hline 9 & 80.00 & 84.38 & Linear & 5 \\
\hline 10 & 85.71 & 71.88 & Quadratic & 5 \\
\hline 11 & 80.00 & 81.25 & Linear & 4 \\
\hline 12 & 87.14 & 90.63 & Quadratic & 4 \\
\hline
\end{tabular}

\subsection{Validity of the model}

The ability of selected model to do classification of new data was examined. Figure 4 shows confusion table of selected model, when checked using entirely different transformer with dissolved gases and oil characteristics measurement data.

\begin{tabular}{|c|c|c|c|c|}
\hline \multirow{3}{*}{ 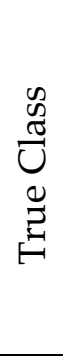 } & Healthy & $\begin{array}{c}94.12 \\
16\end{array}$ & $\begin{array}{c}5.88 \\
1\end{array}$ & $\begin{array}{l}0 \\
0\end{array}$ \\
\hline & Moderate & $\begin{array}{c}8.33 \\
1\end{array}$ & $\begin{array}{c}91.67 \\
11\end{array}$ & $\begin{array}{l}0 \\
0\end{array}$ \\
\hline & Extensive & $\begin{array}{l}0 \\
0\end{array}$ & $\begin{array}{c}33.33 \\
1\end{array}$ & $\begin{array}{c}66.67 \\
2\end{array}$ \\
\hline & & Healthy & Moderate & Extensive \\
\hline
\end{tabular}

\begin{tabular}{|c|c|}
\hline $94.12 \%$ & $5.88 \%$ \\
\hline $91.67 \%$ & $8.33 \%$ \\
\hline $66.67 \%$ & $33.33 \%$ \\
\hline $\begin{array}{c}\text { True } \\
\text { Positive }\end{array}$ & $\begin{array}{c}\text { False } \\
\text { Negative }\end{array}$ \\
\hline
\end{tabular}

Figure 4. Testing confusion table

The model developed is capable of classifying new transformer measurement data with 16 out of 17 transformers in 'Healthy' class correctly classified, 11 out of 12 transformers in 'Moderate' class, and 2 out of 3 transformers in 'Extensive' class.

\subsection{Application of the Model Developed}

The model created has the accuracy of $90.63 \%$ in classifying transformer oil-paper condition to three classes: Healthy; Moderate; and Extensive. This developed model then implemented to 19 transformers data with no furan measurements to do classification of the oil-paper insulation condition. Table 7 shows the classification results which 8 transformers classified as Healthy Transformers, 6 transformers as Moderate Ageing, and 5 transformers as Extensive Ageing.

The classification results were validated using conventional method, such as ratio of $\mathrm{CO} / \mathrm{CO}$, level of $\mathrm{CO}$ and $\mathrm{CO} 2$ respectively, and limit of each oil-characteristics.

Out of dissolved gases parameters, Figure 5 shows that $\mathrm{CO}$ and $\mathrm{CO} 2$ both are caused by overheating of cellulose. Since the focus on this study is condition of oil-immersed paper insulation in transformer, only these two gases were considered correlated, proven by attributes rank in Table 3. The polymeric chains of solid cellulosic insulation (paper, pressboard, wood blocks) contain a large number of anhydroglucose rings, and weak $\mathrm{C}-\mathrm{O}$ molecular bonds and glycosidic bonds which are thermally less stable than the hydrocarbon bonds in oil, and which decompose at lower temperatures. Significant rates of polymer chain scission occur at temperatures higher than $105{ }^{\circ} \mathrm{C}$, with complete decomposition and carbonization above $300{ }^{\circ} \mathrm{C}$. [20]

Ratio of CO2/CO based on IEC60599 [20] is an indicator of the thermal decomposition of cellulose. As the magnitude of $\mathrm{CO}$ increases, the ratio of $\mathrm{CO} 2 / \mathrm{CO}$ decreases. This may indicate an abnormality that is degrading cellulosic insulation [32]. With ratio of $\mathrm{CO} 2 / \mathrm{CO}$ less than 3 , it is generally considered as indication of paper fault with some degree of carbonization [20]. 
Table 7. Assessment of 19 units of $150 \mathrm{kV}$ Power Transformers without Furan measurements data. Based on SVM model developed, the category of oil-paper insulation is predicted. " $\mathrm{H}$ " is for Healthy Transformer, " $\mathrm{M}$ " is for Moderate Ageing, and " $\mathrm{E}$ " is for Extensive Ageing. Green-colored cells show transformers with Healthy class, blue-colored cells show moderate-class transformers, and yellow is transformers with extensive condition. Red-colored cells show parameters in oil which exceeding limits shown in Table 8 and Table 9.

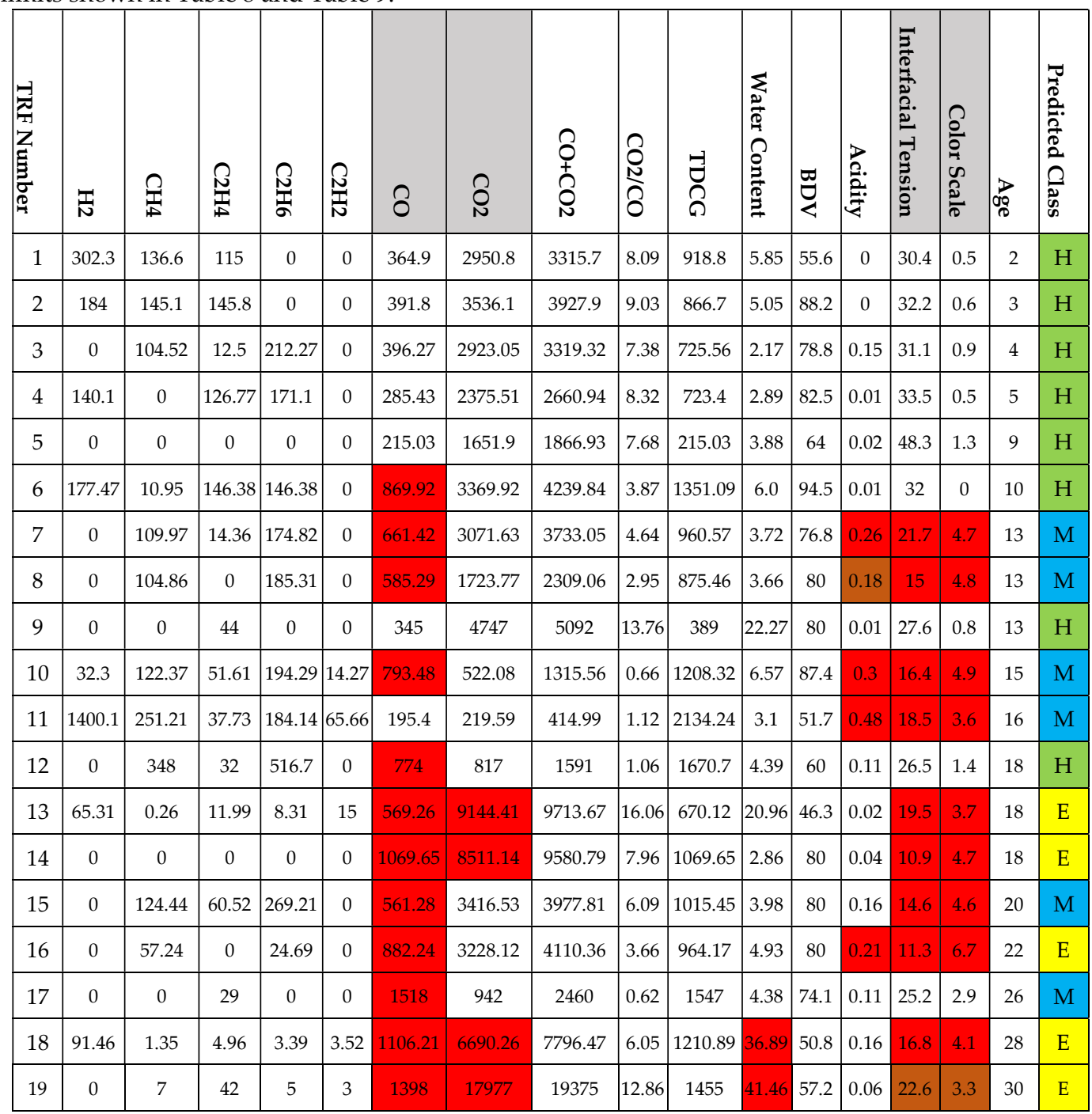

\begin{tabular}{|c|c|c|c|}
\hline 1. & \multicolumn{2}{|c|}{$\mathrm{CO}, \mathrm{CO}_{2}$} & \\
\hline \multirow[t]{4}{*}{2.} & Overheating of oil & \multicolumn{2}{|c|}{ (increasing temperature) } \\
\hline & $\mathrm{C}_{2} \mathrm{H}_{6}$ & $\mathrm{C}_{2} \mathrm{H}_{4}$ & $\mathrm{C}_{2} \mathrm{H}_{4}$ \\
\hline & $\mathrm{CH}_{4}$ & $\mathrm{CH}_{4}$ & $\mathrm{CH}_{4}$ \\
\hline & & & $\mathrm{C}_{2} \mathrm{H}_{2}$ \\
\hline 3. & \multicolumn{3}{|c|}{ Partial discharges (PD) (increasing temperature) } \\
\hline & \multirow[t]{2}{*}{$\mathrm{H}_{2}$} & $\mathrm{H}_{2}$ & \\
\hline & & $\mathrm{C}_{2} \mathrm{H}_{2}$ & \\
\hline 4. & Discharges & & \\
\hline & $\mathrm{C}_{2} \mathrm{H}_{2}, \mathrm{H}_{2}$ & & \\
\hline
\end{tabular}

Figure 5. Principal layout of key-gases characteristic [33], $\mathrm{CO}$ and $\mathrm{CO} 2$ is the main gas indicator of overheating of cellulose in transformer oil. 
Table 8. Significance level of CO and CO2 dissolved in Transformer Oil Insulation based on EPRI Guidelines for the life extensions of substations [34]

\begin{tabular}{lcc}
\hline \multicolumn{1}{c}{ Category } & CO2 $\mathbf{( p p m )}$ & CO $(\mathbf{p p m})$ \\
\hline Condition 1 Normal & $0-2500$ & $0-350$ \\
\hline Condition 2 Modest Concern & $2400-4000$ & $351-570$ \\
\hline Condition 3 Major Concern & $4001-10000$ & $571-1400$ \\
\hline Condition 4 Imminent Risk & $>10000$ & $>1400$ \\
\hline
\end{tabular}

According to [21], transformers $150 \mathrm{kV}$ observed in this study is in Category B, which is power transformers with a nominal system voltage above $72.5 \mathrm{kV}$ and up to and including $170 \mathrm{kV}$. Table 9 shows recommended limits for mineral insulating oils dielectric characteristics.

Table 9. Application and interpretation of dielectric characteristics tests

\begin{tabular}{|l|c|c|c|}
\hline \multirow{2}{*}{\multicolumn{1}{|c|}{ Property }} & \multicolumn{3}{c|}{ Recommended action limits } \\
\cline { 2 - 4 } & Good & Fair & Poor \\
\hline Breakdown voltage (kV) & $>50$ & 40 to 50 & $<40$ \\
\hline Water content (ppm) & $<20$ & 20 to 30 & $>30$ \\
\hline Acidity & $<0.1$ & 0.1 to 0.2 & $>0.2$ \\
\hline Interfacial Tension $($ dyne/cm) & $>25$ & 20 to 55 & $<20$ \\
\hline Color Scale & & & $>3.5$ \\
\hline
\end{tabular}

Transformer number 18 and 19 (TRF \#18 and \#19), which are two of the oldest transformer in these population, classified as E (Extensive Ageing). TRF \#19 shows CO2/CO ratio of 12.86. Ratio more than 10 is an indication of thermal fault in the paper insulation on temperature less than $150^{\circ} \mathrm{C}$, this temperature gives effect to the paper ageing in the long term. TRF \#13 is also shows ratio higher than 10, with high level of $\mathrm{CO}$ and $\mathrm{CO}_{2}$, this also mean TRF \#13 is also undergoing long term ageing in temperature less than $150^{\circ} \mathrm{C}$. Both TRF $\# 18$ and \#19, along with other extensive-classified transformer have high level of $\mathrm{CO}$ and $\mathrm{CO} 2$, exceeding major concern level of $\mathrm{CO}$ and $\mathrm{CO} 2$ concentration in oil shown in Table 8. Besides $\mathrm{CO}$ and $\mathrm{CO} 2$, most of other oil properties of these transformer are at poor condition, such as low interfacial tension and dark oil color. Even, TRF \#18 and \#19 have very high water content, which are up to 36.89 and 41.46 ppm respectively.

At the early stage, TRF \#1 to TRF \#6, which have operating life of 10 years or less, classified as healthy. From oil characteristics point of view, almost all healthy-classified transformer have relatively good oil parameters. This is in line with study in [35], that the ageing process happens during the life of transformer, decrease the condition of the transformer and changes certain parameters in oil insulation.

From the training accuracy, then validated with testing dataset, followed by implementation and comparison with conventional methods and standards, the developed SVM model can successfully classify transformer with no furan measurement and recognize the decreasing trend of transformer oil-immersed paper insulation condition as the operating time increasing.

\section{Conclusions}

Classification analysis of in-service $150 \mathrm{kV}$ Power Transformers insulation condition using Support Vector Machine (SVM) is presented in this article. The proposed method is able to recognize different category of transformer oil-immersed paper insulation condition based on the dissolved gasses and dielectric characteristics measurement data. For training and testing, the measurements data have been divided into two separate datasets. After selecting the best features and cross-validating with different models, the best-performed model has been chosen, resulting in total $90.63 \%$ accuracy in distinguishing the oil-immersed paper insulation condition into three categories: Healthy; Moderate; and Extensive. Further implementation was executed to classify oil-paper condition of Transformers which Furan data is not available, and compared to conventional assessment methods and standards, confirming that the model developed has the ability to do 
classification of current oil-paper condition based on Dissolved Gasses and Dielectric Characteristics. This proved that the model developed has the ability to do prediction of current insulation paper condition category and usefulness to be additional insights in transformer condition monitoring.

Author Contributions: The paper was collaborative effort between the authors. The authors contributed collectively to the theoretical analysis, modelling, simulation, and manuscript preparation.

Acknowledgments: This research was supported by Kementerian Riset, Teknologi, dan Pendidikan Tinggi Republik Indonesia.

Conflicts of Interest: The authors declare no conflict of interest.

\section{References}

[1] J. Unsworth and F. Mitchell, "Degradation of electrical insulating paper monitored with high performance liquid chromatography," IEEE Transactions on Electrical Insulation, vol. 25, no. 4, pp. 737-746, 1990.

[2] L. Cheim, D. Platts, T. Prevost and S. Xu, "Furan analysis for liquid power transformers," IEEE Electrical Insulation Magazine, vol. 28, no. 2, pp. 8-21, 2012.

[3] N. A. Bakar and A. Abu-Siada, "Fuzzy logic approach for transformer remnant life prediction and asset management decision," IEEE Transactions on Dielectrics and Electrical Insulation, vol. 23, no. 5, pp. 3199-3208, 2016.

[4] K. B. Shaban, A. H. El-Hag and K. Benhmed, "Prediction of Transformer Furan Levels," IEEE Transactions on Power Delivery, vol. 31, no. 4, pp. 1778-1779, 2016.

[5] R. A. Prasojo, K. Diwyacitta, Suwarno and H. Gumilang, "Transformer paper expected life estimation using ANFIS based on oil characteristics and dissolved gases (Case Study: Indonesian Transformer)," Energies, vol. 10, no. 1135, 2017.

[6] "Transformer paper condition assessment using adaptive neuro-fuzzy inference system model," in Electrical Engineering and Computer Science, Palembang, 2017.

[7] M. Pal and G. M. Foody, "Feature Selection for Calssification of Hyperspectral Data by SVM," IEEE Transactions on geoscience and remote sensing, vol. 48, no. 5, pp. 2297-2307, 2010.

[8] A. D. Ashkezari, H. Ma, T. K. Saha and C. Ekanayake, "Application of fuzzy support vector machine for determining the health index of the insulation system of in-service power transformers," IEEE Dielectrics and Electricanl Insulation Society, vol. 20, no. 3, pp. 965-973, 2013.

[9] V. Vapnik, The Nature of Statistical Learning Theory, Springer-Verlag, 1995.

[10] "Electric load forecasting based on a least squares support vector machine with fuzzy time series and global harmony search algorithm," Energies, vol. 9, no. 70, 2016.

[11] R. Ayyagari, A. Sivakumar and K. Kannan, "Development of K-Means based SVM regression (KSVMR) technique for boiler flue gas estimation," International Journal on Electrical Engineering and Informatics, vol. 6, no. 2, pp. 359-373, 2014.

[12] B. Wyemeersch, F. De Belie, C. B. Rasmussen and L. Vandevelde, "Classification method to define synchronization capability limits of line-start permanent-magnet motor using mesh-based magnetic equivalent circuit computation results," Energies, vol. 11, no. 998, 2018.

[13] J. Li, Q. Zhang, K. Wang, J. Wang, T. Zhou and Y. Zhang, "Optimal dissolved gas ratios selected by genetic algorithm for power transformer fault diagnosis based on support vector machine," IEEE Dielectrics and 
Electrical Insulation Society, vol. 23, no. 2, pp. 1198-1206, 2016.

[14] W. Mo, T. Kari, H. Wang, L. Luan and W. Gao, "Power transformer fault diagnosis using support vector machine and particle swam optimization," in Computational Intelligence and Design, Hangzou, 2018.

[15] M. Bigdeli, M. Vakilian and E. Rahimpour, "Transformer winding faults classification based on transfer function analysis by support vector machine," IET Electric Power Applications, vol. 6, no. 5, pp. 268-276, 2011.

[16] J. Li, Y. Zhu, Y. Xu and C. Guo, "A transformer fault diagnosis method based on sub clustering reduction and multiclass multi-kernel support vector machine," in Energy Internet and Energy System Integration, Beijing, 2017.

[17] K. Benhmed, K. B. Shaban and A. El-Hag, "Cost effective assessment of transformers using machine learning approach," in IEEE Innovative Smart Grid Technologies Asia, 2014.

[18] I. Gray, "Condition-Based Strategies for Transformer Age Assessment," Transformer Chemistry Services.

[19] Y. Li, M.-J. Tang, F.-J. Wu, G.-J. Zhang, S.-H. Wang and Suwarno, "Aging Assessment of Power Transformer using Multi-Parameters," International Journal on Electrical Engineering and Informatics, vol. 5, no. 1, pp. 34-44, 2013.

[20] "IEC 60599: Mineral oil-impregnated electrical equipment in service - Guide to the interpretation of dissolved and free gases analysis," IEC, 2007.

[21] "IEC 60422: Mineral insulating oils in electrical equipment - Supervision and maintenance guidance," IEC, 2013.

[22] Working Group A2.34, "Guide for Transformer Maintenance," CIGRE, 2011.

[23] N. Das, A. Abu-Siada and S. Islam, "New approach to estimate furan contents in transformer oil using spectroscopic analysis," in Australian Universities Power Engineering Conference , Bali, 2012.

[24] M. Wang, A. Vandermaar and K. Srivastava, "Review of Condition Assessment of Power Transformers in Service," IEEE Electrical Insulation Magazine, vol. 18, no. 6, pp. 12-25, 2002.

[25] B. E. Boser, I. Guyon and V. Vapnik, "A training algorithm for optimal margin classifiers," Proceedings of the Fifth Annual Workshop of Computational Learning Theory, vol. 5, pp. 144-152, 1992.

[26] C. Cortel and V. Vapnik, "Support-Vector Networks," Machine Learning, vol. 20, pp. 273-297, 1995.

[27] Q. Wu and D.-X. Zhou, "SVM Soft Margin Classifiers: Linear Programming versus Quadratic Programming," Neural Computation, vol. 17, pp. 1160-1187, 2005.

[28] C.-W. Hsu and C.-J. Lin, "A Comparison of Methods for Multiclass Support Vector Machines," IEEE Transactions on Neural Networks, vol. 13, no. 2, pp. 415-425, 2002.

[29] V. Vapnik, Statistical Learning Theory, New York: Springer-Verlag, 1998.

[30] W. W. Daniel, Biostatistics: a foundation for analysis in the health sciences, New York: John Wiley and Sons, 1999.

[31] F. Pedregosa, G. Varoquaux, A. Gramfort, V. Michel, B. Thirion, O. Grisel, M. Blondel, P. Prettenhofer, R. Weiss, V. Dubourg, J. Vanderplas, A. Passos, D. Cournapeau, M. Brucher, M. Perrot and E. Duchesnat, "Scikit-learn: Machine Learning in Phyton," JMLR, vol. 12, pp. 2825-2830, 2011.

[32] "IEEE Guide for the Interpretation of Gases Generated in Oil-Immersed Transformers," IEEE Power \& Energy Society, New York, 2008. 
[33] Power Transformers dept. AED Diagnostic Services, "Dissolved gas analysis and supervision of oil condition in transformers and reactors," ABB, Ludvika.

[34] "Guidelines for the Life Extension of Substation Equipment," EPRI, 2008.

[35] K. Diwyacitta, R. A. Prasojo and Suwarno, "Study on Correlation Among Oil Dielectric Characteristics, Dissolved Gases, and Operating Life of $150 \mathrm{kV}$ Power Transformer," International Journal on Electrical Engineering and Informatics, vol. 9, no. 3, pp. 585-603, 2017. 